AIAA 99-3301-CP

\title{
AN ELASTICITY-BASED MESH SCHEME APPLIED TO THE COMPUTATION OF UNSTEADY THREE-DIMENSIONAL SPOILER AND AEROELASTIC PROBLEMS
}

\author{
Robert E. Bartels* \\ Aeroelasticity Branch \\ NASA Langley Research Center \\ Hampton, VA 23681
}

\begin{abstract}
$\underline{\text { Abstract }}$
This paper presents a modification of the spring analogy scheme which uses axial linear spring stiffness with selective spring stiffening/relaxation. An alternate approach to solving the geometric conservation law is taken which eliminates the need for storage of metric Jacobians at previous time steps. Efficiency and verification are illustrated with several unsteady 2-D airfoil Euler computations. The method is next applied to the computation of the turbulent flow about a 2-D airfoil and wing with two and threedimensional moving spoiler surfaces, and the results compared with Benchmark Active Controls Technology (BACT) experimental data. The aeroelastic response at low dynamic pressure of an airfoil to a single large scale oscillation of a spoiler surface is computed. This study confirms that it is possible to achieve accurate solutions with a very large time step for aeroelastic problems using the fluid solver and aeroelastic integrator as discussed in this paper.
\end{abstract}

\section{Introduction}

Aeroelasticity is the study of the interaction of inertial, elastic and aerodynamic forces acting on a structure. Aeroservoelasticity includes the additional mutual interaction of a control system and the aeroelastic structural response. When constructing computational algorithms to model problems in these disciplines, accuracy and robustness must receive due attention. This is especially true in view of recent interest in the accurate computation of fluid-structure interaction in the presence of a strongly nonlinear flow field. This includes examples such as the computation of limit cycle oscillation and the aeroelastic response of a structure due to large-scale control surface motion. To address these issues of robustness and accuracy, recent aeroelastic research has focused on the development of new algorithms that integrate the structural equations of motion in synchronization with the aerodynamic equations of motion Among the methods used or promoted are the closely coupled lagged approach ${ }^{1.2}$ to updating the structure equations and the implicit iterative coupling of both structure and fluid. ${ }^{3}$ Other approaches recently discussed include the Arbitrary Lagrangian-Eulerian (ALE) ${ }^{4.5}$ and the Implicit Continuous-fluid Eulerian (ICED-ALE) methods. ${ }^{6}$ Recent mesh deformation algorithm development has emphasized the robustness and efficiency necessary for coupled structurefluid time marching computations.?

* Aerospace Engineer, Senior Member AIAA

Copyright (c) 1999 by the American Institute of Aeronautics and Astronautics, Inc. No copyright is asserted in the United States under Title 17, U.S. Code. The U.S. Government has a royalty-free license to exercise all rights under the copyright claimed herein for Governmental Purposes. All other rights are reserved by the copyright owner.
The purpose of the present paper is to revisit old approaches to mesh deformation and the integration of the structural equations of motion. In particular the spring analogy mesh scheme is revisited with modifications that enhance its value for structured grids with complex geometry. The finite dimensional state-space predictor-corrector method ${ }^{8}$ also merits renewed consideration in view of recent advances in computational fluid solvers. The performance of the modified spring analogy and the predictor-corrector integrator will be assessed in this paper.

In the development of the present method, several modifications of the typical approach to CFD with a deforming mesh have been made. For unsteady problems, a self-consistent approach to the Jacobian of the coordinate transformation is to compute it by solving an independent equation called the geometric conservation law (GCL) ${ }^{5.9,10}$ In reference 3 , the GCL fluxes are retained in the NavierStokes equations as source terms, replacing the time rate of change of the volume. Not only does this result in selfconsistency, it also eliminates the need for storage of either the discrete time derivative of the Jacobian or of the Jacobian itself at several previous time steps. A variation of that approach is used here. The metric fluxes are retained in the present scheme on both the left and right hand sides of the discrete approximate factorized (AF) equations in a cell centered tinite volume discretization of the equations. In reference 3 , the flow equations were solved in finite difference form using central differencing of the flux terms with explicit and implicit artificial dissipation. Several options are also available in the present code for construction of fluxes and time accuracy. Third order upwind Roe's flux differencing and second order backward time differencing are used here. Subiterations either include the physical time step 
(t-TS subiteration) only or both physical and "pseudo" time step based on local Courant-Friedrichs-Lewy (CFL) number for convergence acceleration ( $\tau$-TS subiteration). This affords the opportunity to assess the effect of the increased robustness of a higher order upwind scheme with pseudo time subiterations on the simulation of unsteady aeroelastic response to a nonlinear flow field. Finally the present deforming mesh scheme is used with a time accurate field equation turbulence model.

Another aspect of this work is the modification of the spring analogy scheme with axial stiffness for use with a structured grid. References 6 and $11-12$ give recent examples of its use with structured and unstructured grids. There are advantages to the spring analogy, most notably its simplicity and ability to smooth grids. However, it does not ensure positivity of control volumes, and in its most typical form is memory intensive. The modified spring analogy using nonlinear torsion stiffness has been shown to preclude non positive control volume (and thus the more restrictive case of grid point crossing).' It does not alone allow spacing control and does not include the smoothing available from the axial spring analogy. Thus it would seem that an optimal approach in the context of the spring analogy would be a combination of several stiffness types. It will be shown that in its present form the spring analogy scheme with linear axial stiffness is efficient and with a structured grid also allows some savings in memory. It can therefore provide a starting point for a more elaborate deforming mesh scheme. It is not, however, without problems. Non positive grid volumes can still be encountered. A partial solution is presented that is useful for at least mildly complex geometry, but it must be emphasized that the present work is considered the initial step in the development of a robust deforming mesh scheme. The intention is that future developments will include the capability of grid adaptation and torsion stiffness that will allow more complex geometry and control over grid orthogonality.

The scheme is first verified for several unsteady 2-D airfoil Euler computations and several unsteady oscillating spoiler Navier-Stokes computations using the Spalart-Allmaras turbulence model. The oscillating airfoil and spoiler computations explore time accuracy and geometric modeling issues. Among other features, the oscillating spoilers are modeled using rigid body rotation rather than the more common shearing control surface motion. This adds somewhat to the realism of the model. A steady rectangular wing 3-D spoiler case and an unsteady 3-D oscillating spoiler case have been computed and are compared with experimental data from the Benchmark Active Controls Technology (BACT) test. ${ }^{13-15}$ The BACT test data presents steady, unsteady and aeroelastic pressure and flutter onset data from a test of a rectangular NACA 0012 wing conducted in the NASA Langley Transonic Dynamics Tunnel. Data associated with that test is available for an active flutter suppression control system using spoiler and trailing edge control surfaces. This present work is the initial part of an effort to utilize this data for the validation of a CFD, aeroelastic, control system simulation methodology that allows for moderately complex control surface motion.

\section{Thin layer Navier-Stokes equations}

The finite volume thin layer Navier-Stokes code that forms the basis for this new development is CFL3D version 5.0. This code has been used extensively to simulate many unsteady 2 and $3-D$ single and multizonal problems. ${ }^{16-17}$ It includes multigrid and grid sequencing capability and has available many choices for turbulence model. It also has the capability to dynamically oscillate a grid ${ }^{18}$ a feature that will be used in the code comparisons that follow. Since the form of the thin layer Navier-Stokes equations as used here and the solution procedures are not typical, the equations and details of the solution will be presented. In a generalized deforming coordinate system the differential form of the equations can be written

$$
\frac{1}{J} \frac{\partial Q}{\partial t}=-R(Q)
$$

where

$$
\begin{aligned}
& R(Q)=\frac{\partial\left(\hat{F}-\hat{F}_{v}\right)}{\partial \xi}+\frac{\partial\left(\hat{G}-\hat{G}_{v}\right)}{\partial \eta}+\frac{\partial\left(\hat{H}-\hat{H}_{r}\right)}{\partial \zeta} \\
& -Q\left[\frac{\partial}{\partial \xi}\left(\frac{\xi_{1}}{J}\right)+\frac{\partial}{\partial \eta}\left(\frac{\eta_{1}}{J}\right)+\frac{\partial}{\partial \zeta}\left(\frac{\zeta_{1}}{J}\right)\right]
\end{aligned}
$$

and $Q=(\rho, \rho u, \rho v, \rho w, e)$. The inviscid flux and viscous diffusive terms are $(\hat{F}, \hat{G}, \hat{H})$ and $\left(\hat{F}_{v}, \hat{G}_{v}, \hat{H}_{v}\right)$ respectively. Note that the time derivative on the left-hand side of equation 2.1 is not written in strong conservation-law form, and that the last bracketed item in the residual is composed of the grid speed fluxes arising from the GCL. Letting

$$
\hat{F}=\left\{\begin{array}{c}
\rho U \\
\rho U u+p \xi_{x} \\
\rho U v+p \xi_{y} \\
\rho U w+p \xi_{z} \\
(e+p) U-p \xi_{t}
\end{array}\right\}
$$

and constructing the remaining inviscid and diffusive fluxes, the complete equation set is obtained. In integral form, the equations 2.1 can be written

$$
\begin{aligned}
\int_{\tau} \int_{V} J^{-1} Q_{1} d V d \tau & =\int_{\int^{2}} \int_{\partial V} \hat{n} \cdot(\hat{E}-\bar{g} Q) d A d \tau \\
& -\int_{\tau} \int_{V}\left(\nabla \cdot \hat{E}_{v}\right) d V d \tau \\
& -\int_{\tau} \int_{V} Q\left(J^{-1} \nabla \cdot \bar{g}\right) d V d \tau
\end{aligned}
$$

where $\bar{g}$ is the grid speed, $\hat{E}=(\hat{F}, \hat{G}, \hat{H})$ and $\hat{E}_{v}=\left(\hat{F}_{v}, \hat{G}_{v}, \hat{H}_{v}\right)$, while $J^{-I}$ is the ratio of the physical to computational volumes. The fluxes are evaluated at the cell face centers, while volume integrals over the computational cell volumes $V$ are evaluated discretely at control volume centers, making this a cell centered finite volume scheme.

The residual composed of the right hand side of equation 2.3 is linearized about the most recent subiteration $m$ and the resulting equations are approximately factored. The resulting discrete equations take on the form

$$
\begin{aligned}
& {\left[\left\{\left(\frac{1+\phi^{\prime}}{J \Delta \tau}\right)+\left(\frac{1+\phi}{J \Delta t}\right)\right\} \tilde{M}+\delta_{\xi} A^{*}+\Omega\right] \Delta q^{\prime}=} \\
& {\left[\frac{\phi^{\prime} \Delta q^{m-1}}{J \Delta \tau}+\frac{\phi \Delta q^{n-1}}{J \Delta t}-\frac{(1+\phi)\left(q^{\prime \prime}-q^{n}\right)}{J \Delta t}\right] \tilde{M}+R\left(Q^{\prime \prime}\right)}
\end{aligned}
$$




$$
\begin{gathered}
{\left[\left(\frac{1+\phi^{\prime}}{J \Delta \tau}\right)+\left(\frac{1+\phi}{J \Delta t}\right) \tilde{M}+\delta_{\eta} B^{*}+\Omega\right] \Delta q^{\prime \prime}=} \\
{\left[\left(\frac{1+\phi^{\prime}}{J \Delta \tau}\right)+\left(\frac{1+\phi}{J \Delta t}\right)+\Omega\right] \tilde{M} \Delta q^{\prime}}
\end{gathered}
$$

$$
\begin{array}{r}
{\left[\left(\frac{1+\phi^{\prime}}{J \Delta \tau}\right)+\left(\frac{1+\phi}{J \Delta t}\right) \tilde{M}+\delta_{\xi} C^{*}+\Omega\right] \Delta q^{m}=} \\
{\left[\left(\frac{1+\phi^{\prime}}{J \Delta \tau}\right)+\left(\frac{1+\phi}{J \Delta t}\right)+\Omega\right] \tilde{M} \Delta q^{\prime \prime}}
\end{array}
$$

for the residual as defined above and

$$
\Omega=-\left[\frac{\partial}{\partial \xi}\left(\frac{\xi_{t}}{J}\right)+\frac{\partial}{\partial \eta}\left(\frac{\eta_{t}}{J}\right)+\frac{\partial}{\partial \zeta}\left(\frac{\zeta_{t}}{J}\right)\right] \tilde{M}
$$

In the equations above $\tilde{M}=\partial Q / \partial q$, $A^{*}=\partial\left(\hat{F}-\hat{F}_{v}\right) / \partial q$ and so forth. The solution variable is $q=(\rho, u, v, w, p)$. Since the fluxes contained in equation 2.5 and 2.2 (last term) are similar in form to the fluxes of the flow quantities both can easily be computed within the same subroutines. This has required the addition of 20-40 coding lines to each of the inviscid flux subroutines (right and lefthand side terms). Furthermore, the desired first or second order accuracy in time is automatically achieved by making use of the metric time derivatives already being used in the spatial fluxes of conserved flow quantities. Since the volumes at several previous time steps are not stored, very little additional memory and no change in the overall code data structure is required. Using this approach does require that equation 2.5 and the metric fluxes of equation 2.2 be recomputed at each subiteration and added to the equations $2.4 \mathrm{a}-\mathrm{c}$, resulting in what appears to be a modest added computational effort.

The metric fluxes in the new terms are evaluated at cell face centers, and are also differenced in time in a way that is consistent with the temporal differencing of conserved flow variables. This results in consistency between new metric fluxes of equation 2.5, the metric time derivatives in the physical fluxes and the integral of $J^{\prime} Q_{r}$ over the control volume, and finally in a temporally and spatially consistent solution of the GCL. The value of the metric Jacobian at time step $n+l$ is used everywhere in equations 2.4 and 2.5. This and the evaluation of the residual at iterate $m$ implies a time discretization of the volume integral equation 2.3 centered about time steps $n+3 / 2$ and $n+1 / 2$. The equations 2.4 also include local CFL based "pseudo" time stepping ( $\tau$ TS subiteration) for convergence. Depending on the values of $\phi$ and $\phi$, the option of first or second order physical and pseudo time stepping can be exercised. In the present computations the temporal discretization of equation 2.3 is accomplished via second order backward differencing (i.e. $\phi$ $=1 / 2$ ). The discrete inviscid fluxes use third order upwind- biased Roe's flux difference splitting and a min-mod flux limiter. Multigrid is used in the following computations.

CFL3D version 5.0 includes many turbulence models. The turbulence model used in the present computations is the Spalart-Allmaras field equation model, selected here primarily because of its robustness at large time steps. Since the differential equation for eddy viscosity in the SpalartAllmaras model is solved in nonconservation form it does not impose any additional requirements on the metrics due to grid deformation. This approach simplifies the procedure when using unsteady field equation turbulence models, since for unsteady computations no additional treatment of metrics or alteration, other than consistent time differencing, is required by the solution of the turbulence model.

\section{Mesh scheme}

The mesh scheme is a modification of the spring analogy using axial spring stiffness. The approach of Batina can be written

$$
M \ddot{\delta}+C \dot{\delta}+K \delta=0
$$

where $K$ is the spring stiffness matrix, $M$ and $\mathrm{C}$ are the mass and damping matrices and the mesh displacement is given by $\delta$. In this formulation $M=C=0$. The stiffness would be defined by $k_{i j}=I n_{i j}$ where $l_{i j}=\left\|\bar{r}_{j}-\bar{r}_{i}\right\|_{2}{ }^{1 / 2}$ and $i$ and $j$ represent two adjacent nodes. For an unstructured grid, storage of stiffness values based on the current locations of each node pair is required. The spring stiffness would be updated as the mesh deforms. For a structured grid the problem is somewhat simplified. Spring stiffness in the mesh interior can be controlled by the spacing of the appropriate boundary grid points. For instance, if the nodes at $(i, j, k)$ and $(i, j, k+l)$ are considered and if boundary spacing at boundaries $i=1$ and $i=i_{\max }$ are used

$$
k_{l n}=1 / l_{k+1 . k}
$$

where $m=k+l$ designates in this case the volume edge between the $k$ and $k+l$ grid points, and

$$
\begin{aligned}
& l_{k+1, k}=f\left(\left\|\bar{r}_{k+1}-\bar{r}_{k}\right\|_{2}\right)_{i=1}^{-1 / 2}+ \\
& (1-f)\left(\left\|\bar{r}_{k+1}-\bar{r}_{k}\right\|_{2}\right)_{i=i \max }-1 / 2 \\
& f=\left(i_{\max }-i\right) /\left(i_{\max }-1\right) .
\end{aligned}
$$

Since these stiffness values are set at the start of the computations they do not vary in time. They also require storage only for each of the six computational boundaries. Linear spring stiffness allows the possibility of grid point crossings, but in view of the fact that this is a restricted case of the general problem of grid lines crossing, the better solution is the addition of nonlinear torsion stiffness. ${ }^{7}$ This can be added to the present spring stiffness at a future time. Finally, it is commented that the axial stiffness approach used here results in smoothing of the mesh and also allows adaptation based on the flow solution. 
The problem of grid collapse around convex surfaces is handled by selectively increasing/decreasing stiffness based on surface curvature. Stiffness values in two coordinate planes normal to a surface are varied based on surface curvature in the coordinate projection of those planes onto the surface. The final mesh is the weighted combination of the two planar solutions. Take for example a $\xi \eta$-plane solid surface at $k=l$ from which curvature information is required to construct the grid. Mathematically the expression for interior grid point location can be written

$$
\begin{array}{r}
\vec{r}_{i j k}=(1-\varepsilon)\left[f_{1} \sum \vec{k}_{m 1} \vec{r}_{m 1}+f_{2} \sum \bar{k}_{m 2} \vec{r}_{m 2}\right] \\
/\left[f_{1} \sum \bar{k}_{m 1}+f_{2} \sum \bar{k}_{m 2}\right]+\varepsilon P
\end{array}
$$

where

$$
f_{1}=e^{C_{1} \Lambda_{\xi}} \quad, \quad f_{2}=e^{C_{1} \Lambda_{\eta}} .
$$

In equation $3.2 \quad \bar{k}_{m 1}=\frac{1}{2} k_{m} e^{-C_{1} \Lambda_{s}}$

$\bar{k}_{m 2}=\frac{1}{2} k_{m} e^{-C_{1} \Lambda_{n}}$ for the volume edges including the endpoints $k+1$ and $k-1$, and $\bar{k}_{m 1}=\bar{k}_{m 2}=k_{m}$ otherwise. The index $m l$ in the summation ranges over the grid points adjacent to $i j k$ in the $\xi \zeta$-plane (indices $j, k$ ) and index $m 2$ in the $\eta \zeta$-plane (indices $i, k$ ). The constant $C_{l}$ is a gain factor that adjusts sensitivity to surface curvature. A value of $C_{i}=20$ seems to work well for many geometries. The surface curvature parameters $\Lambda_{\xi}$ and $\Lambda_{\eta}$ can be arrived at by considering, for example, the surface grids of Figure 1 in the $\xi(j$ index) direction. A measure that accounts, at grid $i, j, k$. for convex or concave curvature at the surface point $i, j$ and $k=l$ is

$$
\Lambda_{\xi}=\frac{\sin 6}{1-\cos 6}
$$

In terms of grid geometry this is

$$
\begin{aligned}
& \Lambda_{\xi}=\Delta \bar{r}_{i} \cdot\left(\Delta \vec{r}_{j+} \times \Delta \vec{r}_{j-}\right) /\left|\Delta \vec{r}_{i}\right|\left(\left|\Delta \vec{r}_{j+}\right|\left|\Delta \vec{r}_{j-}\right|-\Delta \vec{r}_{j+} \cdot \Delta \vec{r}_{j-}\right) \\
& \Lambda_{\eta}=\Delta \vec{r}_{j} \cdot\left(\Delta \vec{r}_{i+} \times \Delta \vec{r}_{i-}\right) / \Delta \vec{r}_{j} \mid\left(\left|\Delta \vec{r}_{i+} \| \Delta \vec{r}_{i-}\right|-\Delta \vec{r}_{i+} \cdot \Delta \vec{r}_{i-}\right)
\end{aligned}
$$

where

$$
\begin{array}{ll}
\Delta \vec{r}_{i}=\vec{r}_{i+1}-\vec{r}_{i-1} & , \quad \Delta \vec{r}_{j}=\vec{r}_{j+1}-\vec{r}_{j-1}, \\
\Delta \vec{r}_{j+}=\vec{r}_{j+1}-\vec{r}_{j} \\
\Delta \vec{r}_{i-}=\vec{r}_{i-1}-\vec{r}_{i} \quad, \quad \text {, Etc. }
\end{array}
$$

In practice the values of $\Lambda_{E}$ and $\Lambda_{\eta}$ are limited in upper and lower bound, currently $\Lambda_{\xi}=\min \left(C_{1} \Lambda_{\xi}, 0\right) / C_{1}$ or $\Lambda_{\xi}=\max \left(C_{1} \Lambda_{\xi},-\right.$ 12)/ $C_{1}$ and similarly for $\Lambda_{\eta}$. An update of $f_{1}\left(\Lambda_{\xi}\right)$ and $f_{2}\left(\Lambda_{\eta}\right)$ are required once per time step for each surface or grid edge plane. This leaves only the computation of equation 3.2 to solve for the interior of the mesh. This is a linear equation typically requiring 1-2 iterations of a Gauss-Seidel procedure for moderate motions of the grid. Finally, the function $P$ defines an outward normal to the surface. Setting $\varepsilon=\exp \left(-C_{2} k\right)$ ensures that at least several grid points near the surface remain orthogonal to the surface.

\section{Aeroelastic method}

The time marching simulation of the aeroelastic responses is obtained using the finite dimensional state space predictorcorrector method, described in references 8 and 19-20, that solves the de-coupled modal structural equations of motion. Several versions of the finite dimensional state space variable method are presented in those references. Of those the predictor-corrector method offers the best performance. The predictor step marches the structure using the solution of the modal equations at step $n$ to get the surface deflection at time step $n+1$. This solution is based on a second order (trapezoidal) extrapolation of the generalized aerodynamic forces at $n$ and $n-l$. This provides the surface shape for a recomputation of the fluid mesh and the fluid domain solution at $n+1$. After the solution of the fluid domain, the corrector step then solves the modal equations at the time step $n+I$ using the averaged generalized forces at $n$ and $n+1$.

\section{$\underline{\text { V. Results }}$}

The first computational results illustrate robustness and efficiency. A comparative study was made for a pitching airfoil using the present deforming mesh scheme in which only the inner boundary is oscillated and that in which the entire grid was dynamically oscillated. The capability to dynamically oscillate the (rigid) grid has been developed and verified in a separate paper. ${ }^{18}$ Both mesh movement schemes used the same grid having dimensions $297 \times 105$. Test cases were the AGARD NACA 0012 cases 3 and 5.21 In each, the number of subiterations and the $\tau$-TS local CFL number were adjusted to give a convergence of the $\mathrm{L}_{2}$-norm of at least $10^{-7}$ at each time step. The results are virtually indistinguishable, as seen in Figure 2 for the Euler computation of AGARD Case 5. This is true even at 16 t.s. per cycle, for which $\Delta t=3.2$ (nondimensionalized by speed of sound), requiring between 20-30 subiterations and some tuning of the local CFL number to adequately converge. An additional test of the time step convergence using the deforming mesh capability is also shown in Figure 2. The lift coefficient shows no discernable variation, while the moment coefficient, although overall nicely convergent, reveals an anomalous lack of convergence around zero degrees (upward motion). At 128 time steps per cycle, any difference in the deforming mesh and rotating frame solutions is imperceptible with the exception of moment coefficient on the downward side of its motion at around -2 degrees. (Figure 3 ) In Figure 4 a comparison of Navier-Stokes solutions at 64 and 2048 time steps per cycle of airfoil oscillation for AGARD case 3 is presented. This case involves slight trailing edge separation. This, and all the computations to follow, are made with the Spalart-Allmaras turbulence model. As can be seen in the figure, very slight differences between the 64 t.s./cycle solution and that at 2048 t.s./cycle only show up during trailing edge separation just after the start of pitch down.

The preceding Euler computations afford the opportunity to assess performance. The grid deformation scheme, the added terms for volumetric change in the Euler equations and the recomputation of cell volumes combined add slightly less than 4 percent CPU time (128 t.s./cycle case, 6 subiterations) over that of the unsteady code with rotating frame. This is with the grid, volumes and metrics updated at each time step. The percent of CPU time is, of course, reduced if larger time 
steps and more subiterations are used. Total code performance is approximately $11.83 \mu \mathrm{sec} /$ cell/iteration C-90 time for the two-dimensional Euler computations.

The final results of this paper are aimed at assessing what is required to compute steady and unsteady two and three dimensional spoiler flows. Specifically, the issues addressed are the geometric modeling of 2-D and 3-D spoilers and the convergence and time step behavior of the present fluid and aeroelastic solvers. In each of the following cases the airfoil/wing-spoiler geometry are based on that used in the Benchmark Active Controls Technology (BACT) NACA 0012 test. $^{13-15}$ This allows a comparison of the present results with the BACT experimental database of oscillating spoiler cases. The spoiler is modeled here as a ramp and backward facing step. A two dimensional version is shown in Figure 5. The three dimensional version, seen in Figure 7 , is modeled as a backward facing wedge having finite spanwise width. As can be seen in both figures, the backward step has a slight slope modeled by spacing three grid points between the spoiler trailing edge and the wing surface. This clearly does not model the cavity beneath the spoiler, nor the gap between the spoiler and flap leading edge. As the spoiler moves the actual spacing over the backward step is constrained via arc length to remain more or less true to the original grid spacing over the wing. Spoiler motion is modeled with rigid body rotation about the spoiler hinge line. The typical approach to modeling the spoiler deflection is to shear the wing surface grids or, at best, to use a two coordinate modal control surface motion model, either of which result in surface warping. The present approach embodies true control surface motion.

Before comparing computed results with the steady and unsteady experimental data, unsteady Navier-Stokes solutions (and grids for a 2-D configuration) are presented in Figures 5 and 6 . This computation investigates time step convergence, which is important before launching into the more costly 3-D oscillating spoiler computations. These computations are especially challenging since they involve the growth and collapse of a large region of recirculating flow. They require significantly more subiterations $(50-60$ at 64 time steps per cycle) and tuning of the $\tau$-TS CFL (for extremely large physical time steps) to reach reasonable accuracy at each time step. Nevertheless, the convergence at each time step was carefully monitored. The $\mathrm{L}_{2}$-norm was maintained at approximately $10^{-7}$ or better at each time step. As can be seen in Figure 6, there is no discernable difference in the lift and moment coefficients in going from 1024 to 64 time steps per cycle of spoiler oscillation. However, the outof-phase part of the transform of the unsteady pressure coefficient shows some reduction in accuracy for the larger time steps. While all the computations appear to be sufficiently accurate for engineering purposes, it is clear that for high accuracy, 260-300 t.s./cycle or more are required. This is true, say, for simulation of response to control system input at a condition near flutter onset, which is typically sensitive to the phase angle of the aerodynamic forcing.

A comparison of steady and unsteady computed results will now be made with experimental data. The purpose here is to assess the computational model for a surface geometry having moderate complexity. The cases computed are for both a 3-D steady and oscillating spoiler on the rectangular NACA 0012 wing used in the Benchmark Active Controls Technology (BACT) Test. As discussed earlier, surface geometry and grids are shown in Figure 7. The deflection in the steady case is $\delta_{s p}=15$ degrees. In all the other computations viscous terms were included only in the direction normal to the solid surface. It was found after the fact that the flow around the trailing edge of the spoiler is moderately altered when streamwise viscous terms are included. For this reason the steady 3-D spoiler calculation included viscous terms in all three index directions. Resolving the grid at the trailing and spanwise edges of the spoiler and the addition of the streamwise viscous terms results in a better match with experiment near the spoiler trailing edge.

The grid for the steady case has somewhat finer resolution than that used in the unsteady 3-D computations. It has dimensions $201 \times 73 \times 73$. Steady pressure coefficients at span locations of $40 \%$ and $60 \%$ are shown in Figure 8. These are just inboard and at mid span of the spoiler which has spanwise edges at $45 \%$ and $75 \%$ span. The pressure distribution in the region of the spoiler and its wake show quite good agreement with experiment. One minor exception is at $60 \%$ span where a slight divergence is observed just ahead of the spoiler trailing edge and just ahead of the shock. The solution at the $40 \%$ span location is off somewhat from $.6 c$ to $.75 c$. This may be due to an inadequate geometric modeling of the spoiler side edges resulting in an improper side wash and vortex development. Figure 9 compares the computed real and imaginary unsteady pressure coefficients for an oscillating spoiler case with experiment. The grid used for this computation was $153 \times 53 \times 41$ in dimension. The mean spoiler angle is 5 degrees and the amplitude of motion is 4.5 degrees. This was computed with 128 t.s./cycle for a reduced frequency based on semi-chord of $k=0.1088(9.56$ $\mathrm{Hz}$. An obvious notable difference between the computations and experiment is observed in the real part of the unsteady pressure coefficient at $60 \%$ span. While not verified, experience with steady cases indicates that some of this difference may be due to the thin-layer viscous approximation used. The other significant difference is in the imaginary part of the unsteady pressure coefficient around the trailing edge of the spoiler at $60 \%$ span.

The simulation of an aeroelastic transient induced by a single oscillation of a 2-D spoiler for an airfoil subject to pitch and plunge is presented in Figures 10-12 and Tables 1 and 2 . This case provides a nominal test of the performance of the complete fluid-mesh-structure solution technique. The aeroelastic response is due to a single large-scale asymmetric oscillation of the spoiler. The spoiler was oscillated at approximately the free vibration frequency of the plunge mode. The dynamic pressure $\left(q_{\infty}=20 p s j\right)$ is low enough to give a moderate aeroelastic response for this size of spoiler motion. After an aeroelastic response using the mesh shown in Figure 5 was computed, it was found that the aeroelastic transient response was sensitive to grid spacing asymmetry between the upper and lower surfaces. A mesh that is symmetric and has the same spacing over both the upper and lower surface was used as the final grid. This grid had dimensions $319 \times 105$. Furthermore, the quality of the grid especially around the spoiler region was found to considerably impact the time step convergence of the aeroelastic solutions. After some effort at improving grid quality, the described solutions were obtained.

The solutions of Figure 10 give transient pitch and plunge response calculated with four time step sizes. Note that the maximum CFL number for the largest time step $(\Delta t=.5859)$ is approximately $2 \times 10^{6}$. The convergence of the plunge mode response with time step size reduction can be clearly seen in the detailed inset figure. Table I summarizes data from these runs. The fluid domain subiterations shown in this table are what were required to converge the solution ( $L$, norm of density) to around $10^{-7}$ at each time step. Between 2048 and 8192 t.s./plunge cycle there is virtually no difference in the 
damping ratios. Between the largest and smallest time steps the difference is less than $1 \%$. The convergence of the aeroelastic response with number of subiterations at the largest time step can be seen in Figure 11. It appears from this figure that 32 subiterations of the fluid domain per time step represent a very nearly converged solution. One can also see from this figure and from Table 2 that if less accuracy is required, fewer subiterations will also result in a solution. On the other hand, a computation that was performed using only a physical time step (t-TS subiteration) was unstable for time steps larger than $\Delta t=.001$. In fact, the solution at $\Delta t=.0012$, shown in Figure 12, diverged shortly after the final time step shown. Even if it had not diverged, this small time step makes this problem solution infeasible with the t-TS method, and necessitates CFL based local time step subiteration (identified as $\tau$-TS in the figure). The accuracy of the t-TS solution is clearly not established with only one computation. However, if the solutions with $\tau$-TS subiterations are converging to the correct solution, as appears from the figure, the $t-T S$ solution even at $\Delta t=.001$ does not appear to be more accurate than the $\tau$-TS solution at a time step 100 times larger.

\section{Concluding remarks}

A numerical scheme for the Euler and Navier-Stokes equations in a general dynamically deforming coordinate system that incorporates the geometric conservation law into the governing equations has been evaluated in this paper. By casting the equations in the form shown here, a somewhat more economical scheme is obtained for problems requiring deforming meshes. A structured mesh scheme, based on the spring analogy, has been applied to moderately complex surface geometry. Its performance has been assessed for time steps spanning a large range, applied to problems from oscillating two-dimensional airfoils and spoilers to a wing having a three-dimensional oscillating spoiler surface. The spoilers have been modeled as a ramp and backward step and the oscillation by true rigid body rotation. The mesh scheme does not appear to result in mesh entanglement for the geometry and mesh motion considered here even with extremely large time step $\left(C F L \approx 2 \times 10^{6}\right)$. This is due both to the fact that the fluid mesh near the moving surface undergoes motion prescribed by the surface and to the selective relaxing/stiffening of the spring stiffness in the grid deformation scheme. Even at very large time steps the scheme appears to retain considerable accuracy if a sufficient number of $\tau$-TS subiterations are used. In the case of strongly nonlinear flow driven by spoiler oscillation, numerical results have shown that the error due to increasing time step that does arise is mainly in the out-of-phase component. This has implications for the accurate computation of flutter onset and other aeroelastic phenomena. The steady and unsteady computed results for the three-dimensional spoiler and wing qualitatively compare well with experiment, with the exception of the fairly small out of phase component of the unsteady pressure coefficient. Finally, aeroelastic capability has been demonstrated for the damped transients of a wing having pitch and plunge modes. The case computed was at low dynamic pressure $\left(q_{\infty}=20\right.$ $p s f$ ) in which the modes were initially perturbed by a single large-scale oscillation of the spoiler surface. The results show that accuracy at very large time step sizes, similar to that used in the oscillating spoiler computations, can be achieved here as well. This suggests that for these cases and solution method, the convergence of the fluid solution is the dominant factor in the accuracy of the structure-fluid solution. Again, this is possible because of the r-TS flow field solution. Because of stability limitations, the largest time step possible with t-TS subiteration was at least 600 times smaller than the largest time step with $\tau$-TS subiteration.

\section{References}

1. Schuster, David M., Vadyak, Joseph,. Atta, Essam, "Static Aeroelastic Analysis of Fighter Aircraft Using a Three-Dimensional Navier-Stokes Algorithm." Joumal of Aircraft, Vol. 27, No. 9, pp. 820-825, (1990).

2. Guruswamy, G. P., "Unsteady Aerodynamic and Aeroelastic Calculations for Wings Using Euler Equations." AIAA Joumal, Vol. 28, No. 3, pp. 461-469, (1990).

3. Melville, R. B., Morton, S. A., Rizzetta, D. P., "Implementation of a Fully-Implicit, Aeroelastic Navier-Stokes Solver." AIAA paper 97-2039, AIAA 13th Computational Fluid Dynamics Conference, June 29-July 2, 1997. Snowmass Village, CO.

4. Bendiksen. O. O., "A new approach to computational aeroelasticity." AIAA Paper 91-0939.

5. Farhat, Charbel, Lesoinne, Michel, Maman, Nathan, "Mixed Explicit/Implicit Time Integration of Coupled Aeroelastic Problems: Three-Field Formulation, Geometric Conservation and Distributed Solution," Intemational Joumal for Numerical Methods in Fluids, Vol. 21, pp. 807-835, (1995).

6. Vahdati, M., Imregun, M., "An application of the ICED-ALE methodology to integrated non-linear aeroelasticity analyses." Journal of Engineering Computations, Vol. 14, No. 3, pp. 281-305, (1997).

7. Farhat, C., Degand, C., Koobus, B., Lesoinne, M., "An Improved Method of Spring Analogy for Dynamic Unstructured Fluid Meshes." AIAA 1998 Stuctural Dynamics and Materials Conference, April 20-23, 1998, Long Beach, CA.

8. Edwards, J. W., Bennett, R. M., Whitlow, W. Jr., Seidel, D. A., "Time-Marching Transonic Flutter Solutions Including Angle-of-Attack Effects," Joumal of Aircraft, Vol. 20, pp. 899-906, (1983).

9. Thomas, P. D., Lombard, C. K., "Geometric Conservation Law and Its Application to Flow Computations on Moving Grids." AIAA Journal, Vol. 17, pp. 1030-1037, (1978).

10. Batina, John T., "Unsteady Euler Airfoil Solutions Using Unstructured Dynamic Meshes," AIAA Paper 89-0115.

11. Lee-Rausch, E. M., Batina, J. T., "Wing Flutter Boundary Prediction Using Unsteady Euler Aerodynamic Method." Journal of Aircraft, Vol. 32, pp. 416-422, (1995).

12. Blom, F. J., Leyland, P., "Analysis of Fluid-Structure Interaction by Means of Dynamic Unstructured Meshes," 4th International Symposium on FluidStructure Interactions. Aeroelasticity, Flow-Induced Vibration and Noise, Volume 1, ASME, 1997, pp. 3-10.

13. Scott, Robert C., Hoadley, Sherwood T., Wieseman Carol D., Durham, Michael H., "The Benchmark Active Controls Technology Model Aerodynamic Data," AIAA paper 97-0829.

14. Bennett, R. M., Eckstrom, C. V., et al., "The Benchmark Aeroelastic Models Program - Descripion and Highlights of Initial Results." NASA TM-104180. Dec. 1991.

15. Waszak, Martin R., "Modeling the Benchmark Active Control Technology Windtunnel Model for Application to Flutter Suppression." AIAA paper 96-3437-CP, AIAA Atmospheric Flight Mechanics Conference.

16. Rumsey, C., Sanetrik. M., Biedron, R., Melson, N., Parlette, E., "Efficiency and Accuracy of TimeAccurate Turbulent Navier-Stokes Computations." 
Computers and Fluids, Vol. 25, No. 2, pp. 217-236, (1996).

17. Rumsey, Christopher L., Vatsa, Veer N., "Comparison of the Predictive Capabilities of Several Turbulence Models." Journal of Aircruft, Vol. 32, No. 3, pp. 510514. (1995).

18. Anderson, W. Kyle, Thomas, James L., Rumsey, Christopher L., "Extension and Applications of FluxVector Splitting to Unsteady Calculations on Dynamic Meshes." AIAA Paper 87-1 152-CP, 1987.

19. Cunningham, Herbert J., Batina, John T., Bennett, Robert M., "Modern Wing Flutter Analysis by Computational Fluid Dynamics Methods." Journal of Aircraft, Vol. 25, pp. 962-968, (1988).
20. Rausch, R. D., Batina, J. T., Yang, H. T. Y., "Euler Flutter Analysis of Airfoils Using Unstructured Dynamic Meshes." Journal of Aircruft, Vol. 27, pp. 436-443, (1990)

21. Compendium of Unsteady Aerodynamic Measurements, AGARD Report No. 702, August 1982.
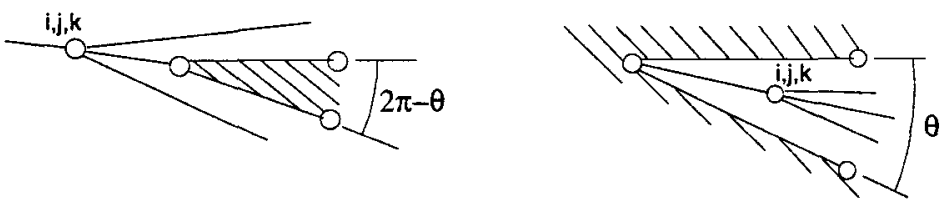

Figure 1. Orientation of surface and interior grids.
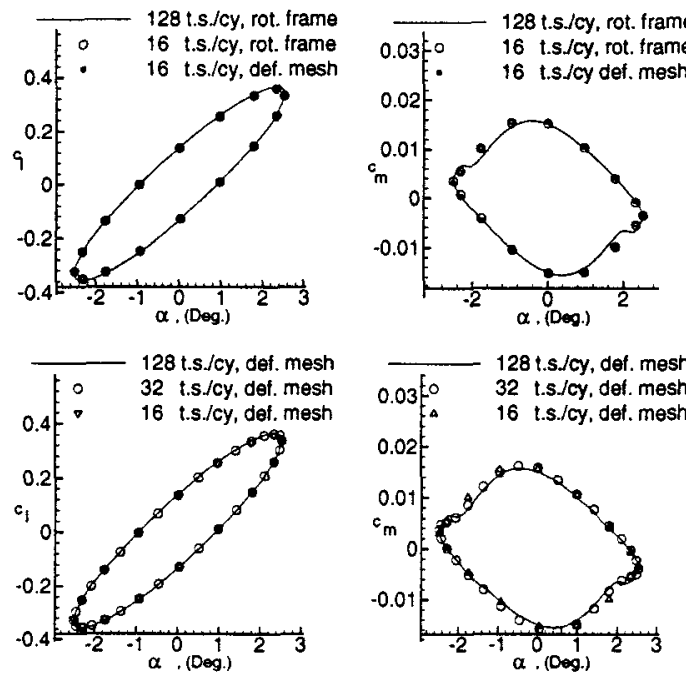

Figure 2. Time step study for deforming mesh scheme. AGARD NACA 0012 Case 5, NACA 0012, $\mathrm{M}_{\infty}=0.755$,

$\alpha=0.016+2.51 \sin \left(2 \mathrm{ktM}_{\infty}\right)$ degrees, $\mathrm{k}=0.081$ 

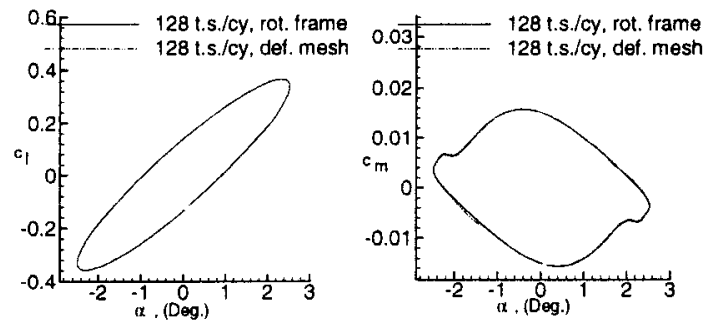

Figure 3. Comparison of results for rotating frame and deforming mesh. AGARD NACA 0012 Case 5, NACA 0012, $\mathrm{M}_{\infty}=0.755$, $\alpha=0.016+2.51 \sin \left(2 \mathrm{ktM}_{\infty}\right)$ degrees, $\mathrm{k}=0.081$
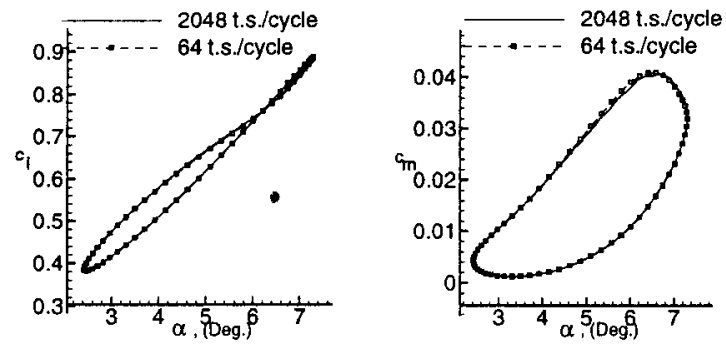

Figure 4. Time step study for deforming mesh viscous computation. AGARD NACA 0012 Case $3, \mathrm{M}_{\infty}=0.60, \alpha=4.86+2.44 \sin \left(2 \mathrm{ktM} \mathrm{M}_{\infty}\right)$ degrees, $\mathrm{k}=0.081$
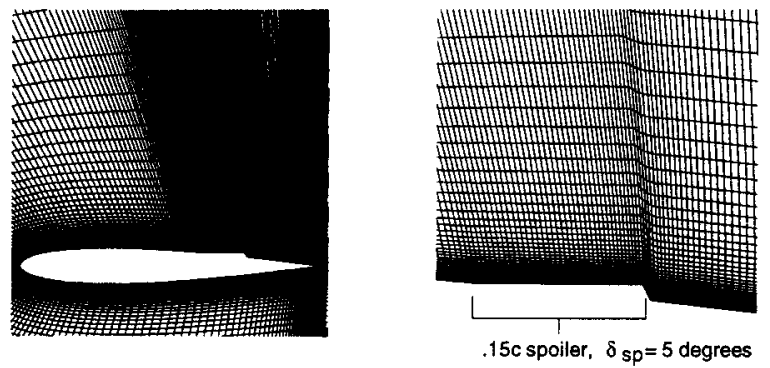

Figure 5. Two-dimensional mesh for oscillating spoiler study.
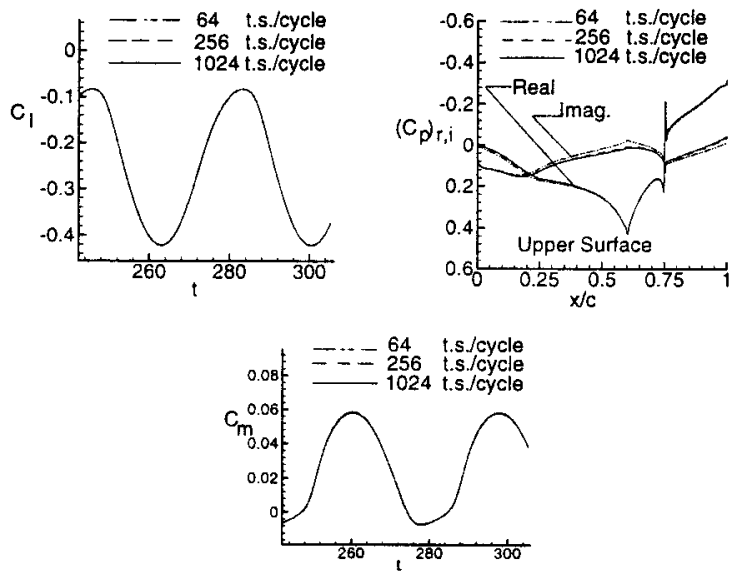

Figure 6. Time step study for 2D NACA 0012 airfoil with oscillating spoiler. $M_{\infty}=0.77, \alpha=0$ degrees, $\delta_{\mathrm{sp}}=5+4.5 \sin \left(2 \mathrm{M}_{\infty} \mathrm{kt}\right)$ degrees. 


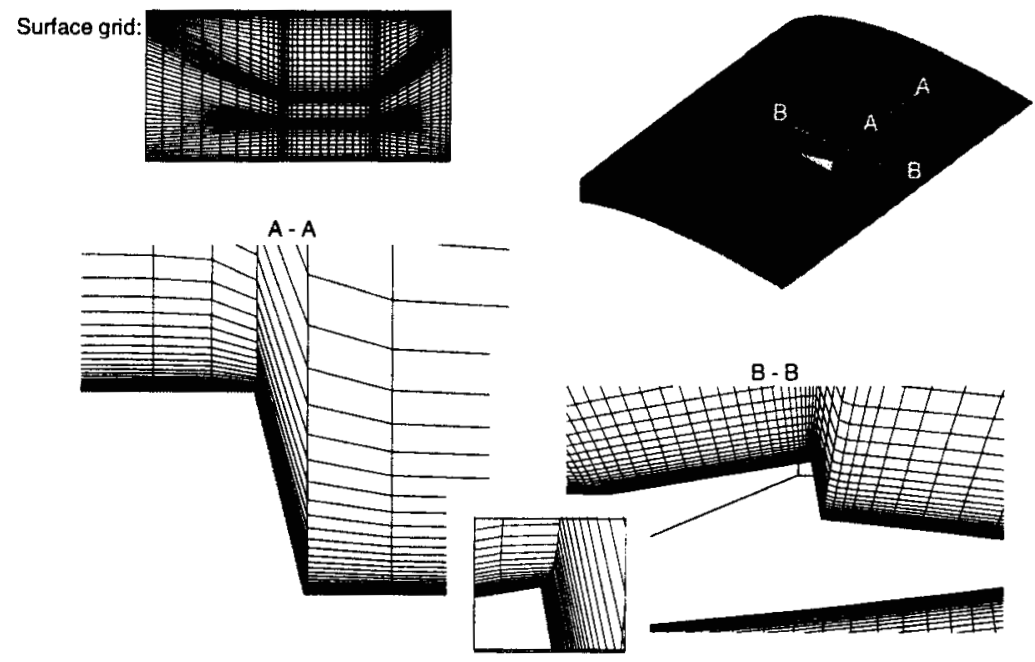

Figure 7. BACT model upper surface and mesh.

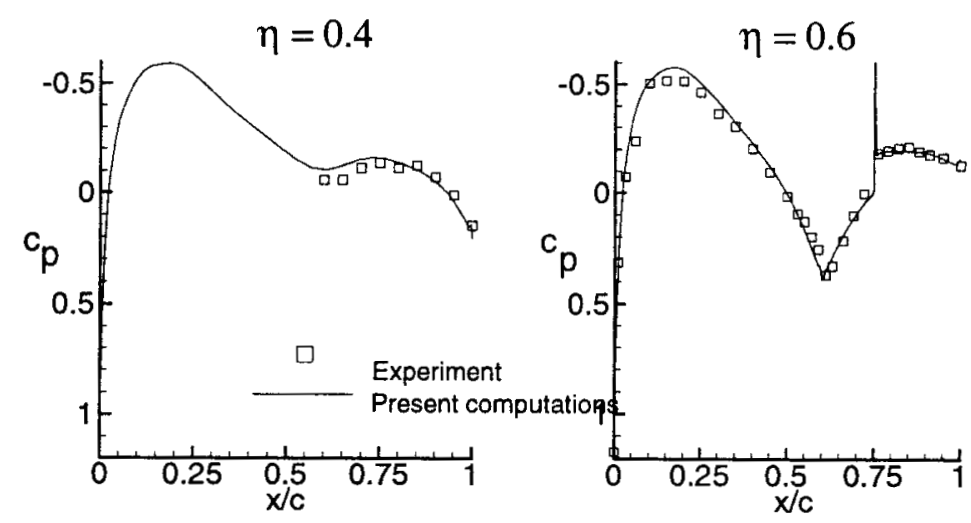

Figure 8. Comparisons of BACT model steady pressure coefficients. $M_{\infty}=0.77, \alpha=0$ degrees $\delta_{\mathrm{sp}}=15$ degrees. 

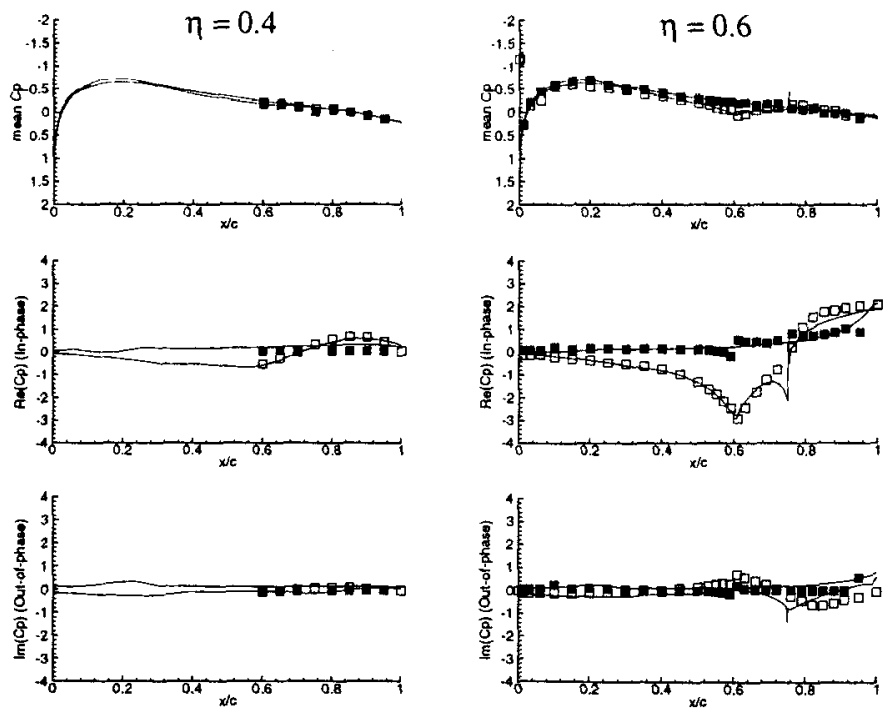

- Experiment, upper surface

Experiment, lower surface

Present computations

Figure 9. Comparisons of BACT model unsteady pressure coefficients. $M_{\infty}=0.77, \alpha=0$ degrees $\delta_{s p}=5+4.5 \sin \left(2 M_{\infty} k t\right)$ degrees

$\mathrm{h} / \mathrm{c}, \alpha$

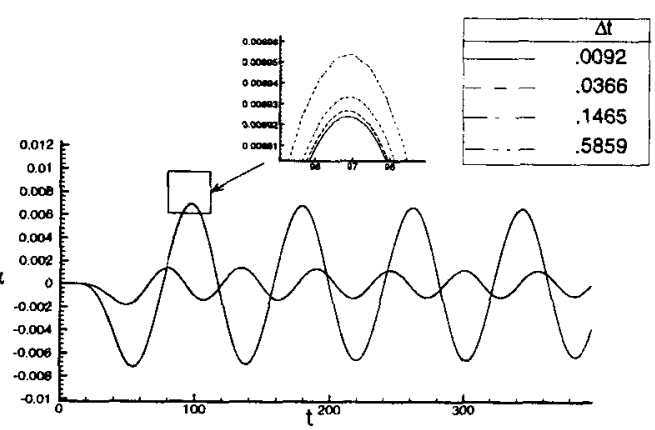

\begin{tabular}{|c|c|c|c|c|}
\hline$\Delta t$ & $\begin{array}{c}\text { time steps } \\
\text { (per plunge } \\
\text { cycle) }\end{array}$ & $\begin{array}{c}\text { No. of fluid } \\
\text { domain } \\
\text { sub-iterations }\end{array}$ & $\xi_{h}$ & $\xi_{\alpha}$ \\
\hline 0.0092 & 8192 & 2 & .02781 & .05229 \\
0.0366 & 2048 & 6 & .02781 & .05230 \\
0.1465 & 512 & 12 & .02779 & .05228 \\
0.5859 & 128 & 32 & .02761 & .05218 \\
\hline
\end{tabular}

Figure 10. Computed aeroelastic response due to a single spoiler oscillation ( 9.5 degree excursion), 2D BACT model. $\mathrm{M}_{\infty}=0.77, \alpha=0$ degrees, $\mathrm{q}_{\infty}=20 \mathrm{psf}, \mathrm{U}_{\infty}=373 \mathrm{fps}$. Figure 10 ).

Table 1. Computed damping ratios for two aeroelastic modes, (Same model and condition as Figure 10). 


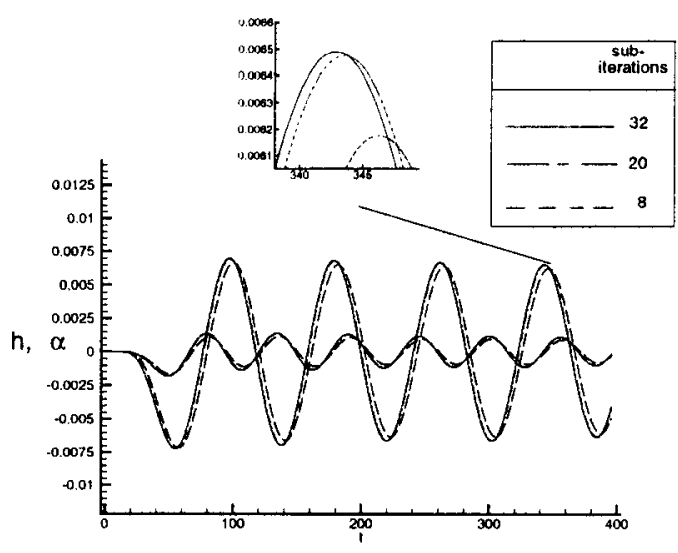

Figure 11. Computed aeroelastic response due to a single spoiler oscillation. (Same model and condition as Figure 10 , $\Delta \mathrm{t}=0.5859$.)

\begin{tabular}{|c|c|c|c|}
\hline$\Delta t$ & $\begin{array}{c}\text { time steps } \\
\text { per plunge } \\
\text { cycle) }\end{array}$ & $\begin{array}{c}\text { No. of fluid } \\
\text { domain } \\
\text { sub-iterations }\end{array}$ & $\xi_{\mathrm{h}}$ \\
\hline 0.5859 & 128 & 32 & .02761 \\
0.5859 & 128 & 20 & .02750 \\
0.5859 & 128 & 8 & .02648 \\
\hline
\end{tabular}

Table 2. Effect of number of subiterations on damping ratio, (Same model and condition as Figure 10)

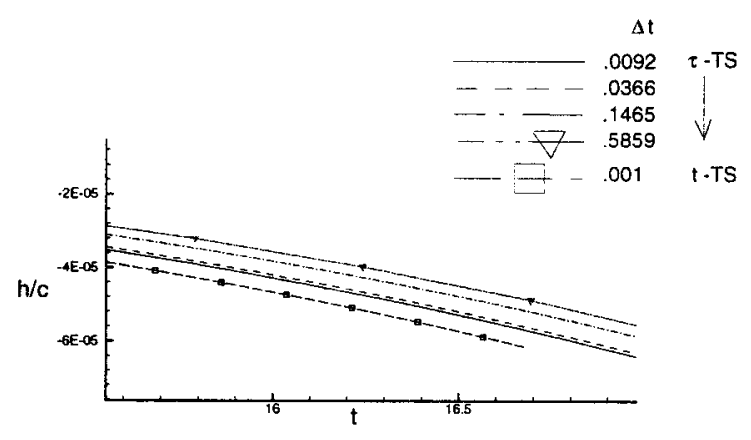

Figure 12. Comparison of t-TS and $\tau$-TS time stepping for aeroelastic response (same model and condition as Figure 10). 